\title{
POLIMEROSSOMOS VERSUS LIPOSSOMOS: A EVOLUÇÃO DA "BALA MÁGICA"
}

\author{
Alexsandra Conceição Apolinário, Juliana de Almeida Pachioni-Vasconcelos, Adalberto Pessoa Jr. e Carlota de Oliveira \\ Rangel-Yagui* \\ Departamento de Tecnologia Bioquímico-Farmacêutica, Faculdade de Ciências Farmacêuticas, Universidade de São Paulo, Av. \\ Prof. Lineu Prestes, 580 - Bloco 16, Cidade Universitária, 05508-000 São Paulo - SP, Brasil
}

Recebido em 29/12/2016; aceito em 08/03/2017; publicado na web em 15/05/2017

\begin{abstract}
POLYMERSOMES VERSUS LIPOSOMES: THE "MAGIC BULLET" EVOLUTION. Polymeric vesicles known as polymersomes are formed by the self-assemble of amphiphilic block copolymers and have structural organization similar to liposomes. However, polymersomes present higher physicochemical stability and structure versatility due to their polymeric nature. In this review we describe the main aspects related to the structure and applications of these systems for drug delivery.
\end{abstract}

Keywords: drug delivery; polymersomes; liposomes; amphiphilic block-copolymers; self-aggregated nanostructures.

\section{INTRODUÇÃO}

As pesquisas com sistemas de liberação de fármacos e biofármacos a partir de nanoestruturas têm revolucionado as ciências farmacêuticas no mundo todo. ${ }^{1,2}$ Estes nanocarreadores (NC) têm propriedades peculiares relacionadas ao tamanho em escala nanométrica e área superficial aumentada, o que lhes confere potencial para modular características farmacocinéticas e farmacodinâmicas de fármacos. Portanto, são capazes de melhorar a estabilidade in vitro e in vivo de fármacos, prolongar o tempo de circulação sanguínea e permitir a liberação controlada e direcionada.,

Devido às vantagens dos $\mathrm{NC}$, a cada dia estudos descrevem novas possibilidades de aperfeiçoar ainda mais estes sistemas e percebe-se uma evolução constante no campo da nanotecnologia aplicada a produtos farmacêuticos, especialmente na oncologia. As pesquisas relatam $\mathrm{NC}$ amplamente usados como nanopartículas poliméricas, nanopartículas lipídicas sólidas, nanopartículas metálicas e lipossomos (LP), com diferentes abordagens referentes à composição, inovações na técnica de produção e alternativas para liberação sítio-dirigida, de modo que haja aprimoramento funcional do produto final..$^{5-9}$

Um exemplo bastante atual de inovação em nanocarreadores são as vesículas poliméricas, descritas inicialmente em 1981 por Kunitake e colaboradores ${ }^{10}$ e em 1995 estudadas com maior embasamento físico-químico por Zhang \& Eisenberg. ${ }^{11}$ Posteriormente, essas vesículas foram denominadas polimerossomos (PL) por Discher e colaboradores, ${ }^{12}$ uma vez que apresentam organização estrutural semelhante aos LP. PL podem ser conceituados como nanocarreadores supramoleculares poliméricos com estrutura vesicular. Enquanto LP são vesículas de interior aquoso compostas por uma membrana lipídica, os PL, que também apresentam interior aquoso, são compostos por uma membrana de copolímeros anfifílicos.

Para compreender a importância dos PL, é válido reforçar que são morfologicamente análogos aos LP, os quais estão entre os NC mais empregados no campo farmacêutico e foram relatados pioneiramente nos anos 60 por Bangham e seus colaboradores, que descreveram estruturas vesiculares organizadas em bicamadas originadas por fosfolipídeos em soluções aquosas. ${ }^{13}$ Após estudos iniciais com LPs, percebeu-se que estes nanocarreadores poderiam se encaixar no conceito proposto por Paul Ehrlich conhecido por "Bala Mágica" ou do inglês "Magic Bullet", conceito descrito em um artigo publicado

*e-mail: corangel@usp.br pela Química Nova em 2002 intitulado "Lipossomas: a bala mágica acertou?". ${ }^{14}$ De acordo com Ehrlich, deveriam haver compostos, e aqui extrapolamos para nanoestruturas, capazes de atingir o alvo biológico sem causar danos ao organismo. Quase 30 anos após estes estudos preliminares, em 1995, a primeira formulação lipossomal, Doxil $^{\circledR}$, foi aprovada pela Food and Drug Administration (FDA) para o tratamento do sarcoma de Kaposi associado à Síndrome de Imunodeficiência Adquirida (SIDA). Atualmente, estima-se que 14 novos sistemas de LP foram aprovados e cerca de 21 encontram-se em ensaios clínicos. ${ }^{15}$ No entanto, a instabilidade físico-química é a principal desvantagem inerente a este NC devido à sua natureza lipídica, o que em geral não ocorre com os PL em razão da estrutura polimérica, que é físico-quimicamente mais estável. Portanto, estes sistemas vêm sendo empregados recentemente em diversas abordagens de estudos para drug delivery. ${ }^{12,16}$

Os conceitos envolvendo os PL abrangem desde a compreensão da estrutura e propriedades dos copolímeros anfifílicos que constituem tais NC, passando pelos métodos de produção e caracterização das vesículas, bem como as principais aplicações. Neste artigo, descrevemos os principais aspectos relacionados a estes tópicos. Cabe ressaltar que não existe ainda na literatura artigos em língua portuguesa introduzindo o conceito de polimerossomos e suas potencialidades no campo farmacêutico.

Além disso, consideramos importante a divulgação de temas na área de nanotecnologia farmacêutica assim como fizeram antes pesquisadores brasileiros, como por exemplo Schaffazick e colaboradores, ${ }^{17}$ ao detalharem os aspectos sobre a formação e caracterização das nanopartículas, Oliveira e colaboradores ${ }^{18}$ em 2004, quando explanaram os principais aspectos da estrutura de microemulsões e Durán e colaboradores, ${ }^{19}$ que descreveram didaticamente a tecnologia de produção de nanocristais de fármacos.

\section{LIPOSSOMOS VERSUS POLIMEROSSOMOS}

Diante da analogia entre LP e PL, surge o questionamento sobre as diferenças, vantagens e desvantagens de um em relação ao outro. A relevância dos LP (Figura 1) dentro da nanotecnologia é indiscutível, são várias aplicações na área farmacêutica que incluem desde liberação de ativos cosméticos até fármacos anticancerígenos..$^{20,21}$ Porém, as pesquisas com PL vêm ganhando espaço nas nanociências, já que são sistemas mais estáveis fisicoquimicamente e mais resistentes mecanicamente em relação às vesículas lipídicas em razão das propriedades químicas dos polímeros que os constituem. ${ }^{22}$ 


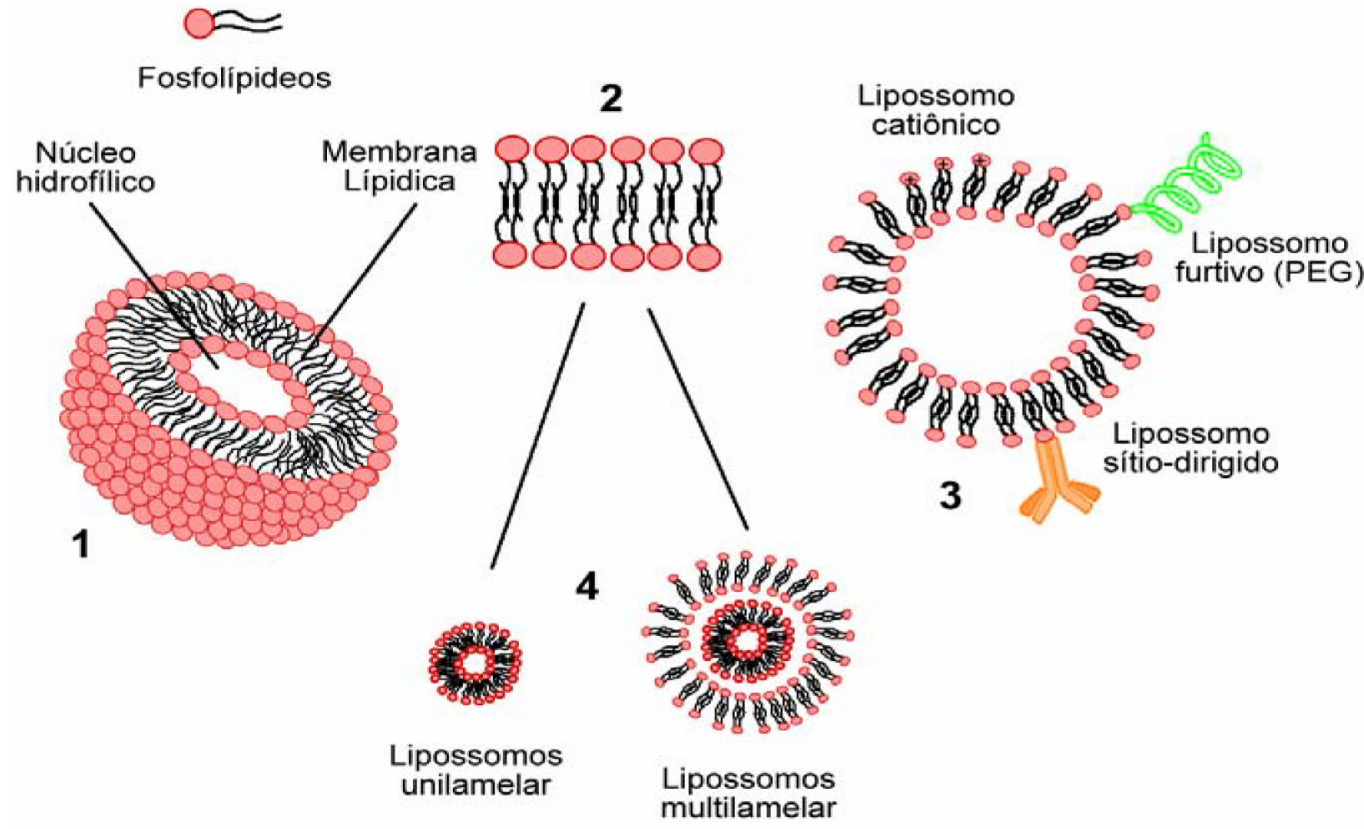

Figura 1. 1) Estrutura dos lipossomos constituídos por fosfolipídeos. 2) Bicamadas lipídicas. 3) Lipossomos com modificações na superfície (peguilados, com carga e com anticorpos) 4) Tipos de lipossomos de acordo com o número de camadas

A composição dos LP consiste geralmente de fosfolipideos e esteróis; estes componentes, juntamente com o método de produção, determinam as propriedades físicoquímicas destes NC. ${ }^{23}$ Algumas características dos LP como taxa de encapsulação, estabilidade da membrana e degradação são difíceis de controlar e estes sistemas apresentam baixa versatilidade química. ${ }^{12}$ Uma outra desvantagem deste sistema diz respeito ao processo de esterilização necessário às formulações de uso intravenoso, que é limitado devido à susceptibilidade dos fosfolipídeos à degradação físico-química. ${ }^{24}$

A maior parte das pesquisas recentes com LP para drug delivery refere-se a sistemas compostos por vesículas unilamelares de 50-150 nm. Essa faixa de tamanho reflete um balanço entre eficiência de encapsulação (aumenta quanto maior o agregado formado), estabilidade dos LP (diminui para tamanhos superiores à faixa ótima de 80-200 $\mathrm{nm}$ ) e habilidade em extravasar o sistema vascular (diminui com o aumento do tamanho). A espessura da membrana é de aproximadamente $4 \mathrm{~nm}$. LP podem apresentar uma camada de polietielnoglicol (PEG) covalentemente ligada à superfície para evitar o reconhecimento pelos macrófagos. Nesses casos são denominados de LP peguilados ou furtivos. Além de PEG, podem também apresentar em sua superfície ligantes com funções definidas, por exemplo para reconhecimento do alvo. ${ }^{25}$

A natureza lipídica torna os LP suscetíveis a processos de degradação como peroxidação lipídica, hidrólise, além da probabilidade de transição de fase $\left(T_{c}\right)$, que é um importante parâmetro a ser avaliado para estes carreadores, já que se trata de um parâmetro que indica mudanças no estado físico da bicamada lipídica, que pode passar de uma conformação ordenada de gel para um estado desordenado líquido-cristalino em resposta à mudanças de temperatura. ${ }^{24}$ Esta propriedade influencia na permeabilidade, fusão, agregação e ligação à proteínas. A incorporação de alguns lipídeos fluidos na bicamada de lipossomos pode diminuir a $T_{c}$, aumentando sua fluidez. ${ }^{26}$

Para os PL, a instabilidade relacionada aos fenômenos de degradação físico-química ou mesmo mecânica diminui em razão das propriedades dos copolímeros anfifílicos que compõe estes NC. Copolímeros anfifílicos são compostos de duas ou mais cadeias homopoliméricas distintas, covalentemente ligadas em um ou mais pontos de junção que se auto-organizam em diferentes morfologias, incluindo micelas ou vesículas poliméricas, quando em contato com solventes, dependendo das condições externas como temperatura, agitação e $\mathrm{pH} \cdot{ }^{27-29}$ Para aumentar a resistência de LPs ao trato gastrointestinal, por sua vez, eles podem ser modificados através da incorporação de sais biliares ou pelo desenvolvimento de LPs multilamelares. ${ }^{30}$

A estabilidade mecânica dos PL é determinada em razão da espessura da bicamada e, portanto, estes sistemas são geralmente mais rígidos do que os LP. Os PL possuem membranas mais espessas (2-20 $\mathrm{nm}$ ) do que os LP (3-5 nm), assim têm a vantagem de tenacidade superior e cinética de liberação mais lenta. ${ }^{29} \mathrm{~A}$ baixa massa molecular dos lipídeos confere fluidez na bicamada dos LP, ${ }^{31}$ enquanto que a espessura da membrana de PL pode ser controlada pela massa molecular dos copolímeros. Quanto maior a massa molecular dos copolímeros mais forte é a interação entre esses na bicamada formada, resultando em PL com membranas mais espessas e mais resistentes. ${ }^{29}$ Consequentemente, os PL apresentam elevada estabilidade mecânica, comportamento elástico e viscosidade de membrana elevada. ${ }^{31}$

As semelhanças entre LP e PL referem-se principalmente à morfologia vesicular, bem como aos métodos de produção, especialmente o de hidratação do filme, que será discutido adiante. Ambas as estruturas são baseadas em princípios termodinâmicos semelhantes, que implicam na formação espontânea de vesículas em razão das características anfifílicas dos componentes básicos (lipídeos ou copolímeros). ${ }^{32}$

\section{COPOLÍMEROS ANFIFÍLICOS}

Copolímeros anfifílicos (CA) são análogos macromoleculares de tensoativos e lipídeos, os quais contêm duas ou mais sequências de homopolímeros quimicamente distintas quanto à polaridade e unidas por ligação covalente. ${ }^{33,34}$ As sequências de homopolímeros são chamadas de blocos, por isso esses copolímeros são denominados copolímeros dibloco (AB), tribloco (ABA) ou até multibloco, dependendo da quantidade de cadeias de homopolímeros. Assim, são capazes formar estruturas versáteis que podem encapsular fármacos hidrofílicos e/ ou hidrofóbicos. Mais de 20 morfologias já foram identificadas a partir da auto-agregação de CA, as quais são termodinamicamente induzidas e/ou cineticamente controladas, destacando-se as micelas e vesículas, que serão discutidas no próximo tópico. ${ }^{35}$ São exemplos 
de copolímeros anfifílicos os copolímeros de poli(óxido de etileno)poli(óxido de propileno)-poli(óxido de etileno), PEO-PPO-PEO; poli(óxido de etileno)-poli(ácido lático), PEO-PLA e poli(óxido de etileno)-policaprolactona (PEO-PCL).

A auto-agregação dos copolímeros anfifílicos depende primordialmente de três parâmetros: a massa molecular média $(\mathrm{Mw})$, a massa ou fração de volume de cada bloco $(f)$ e a energia efetiva de interação entre as moléculas de copolímero. ${ }^{36}$ As propriedades físicas dos PL estão diretamente relacionadas à espessura da membrana, a qual por sua vez depende da massa molecular dos copolímeros; copolímeros anfifílicos de massa molecular mais elevada resultam em PL de membrana mais espessa, robusta e estável. ${ }^{37} \mathrm{~A}$ espessura da membrana dos PL implica diretamente nas seguintes propriedades destes sistemas: taxa de alongamento (Ka), área crítica de tensão $\left(\alpha_{c}\right)$, tensão de lise $(\zeta c)$ potencial de quebra $(\mathrm{Vc})$, flexibilidade $(K \mathrm{~b})$, difusibilidade lateral (D). ${ }^{29}$

CAs sintéticos podem apresentar considerável polidispersão, ou seja, podem conter cadeias de homopolímeros de comprimentos diferentes e, assim, a massa molecular não apresenta um único valor. Os polímeros existem como uma distribuição de comprimentos de cadeia e massas moleculares. Nesse sentido, a Mw deve ser descrita como a soma da média das massas moleculares calculadas para todas as cadeias. Além da Mw, a massa molecular média em número, do inglês average molecular weight (Mn), e índice de polidispersão (PDI) devem ser analisados na escolha dos copolímeros. ${ }^{37}$

Os CAs podem ainda ser constituídos de um bloco flexível (coil) e outro rígido ( $r o d$ ), o qual possui capacidade limitada de esticar e se empacotar durante o processo de auto-organização; esta conformação resulta da $\pi$-conjugação ao longo da cadeia do CA (Figura 2). Dessa maneira, são classificados em copolímeros coil-coil ou rod-coil (Figura 2).$^{38} \mathrm{CA}$ do tipo rod-coil formam estruturas supramoleculares com dimensões na escala nanométrica, que não são comuns em microfases formadas por copolímeros flexíveis como fases cristalinas termotrópica e liotrópicas. ${ }^{39} \mathrm{Na}$ verdade, o bloco rod não tem a mesma entropia conformacional do bloco coil, o que restringe a habilidade para esticar e se auto-organizar. Exemplos de copolímeros rod-coil são estruturas de cadeia inflexível advindas de conjugação $\pi$ (polímeros semicondutores), estruturas secundárias helicoidais (biomoléculas) ou grupos aromáticos; todos levam à adoção de conformações estendida e com cadeia rígida. ${ }^{40}$

\section{Copolímero Rod-Coil}

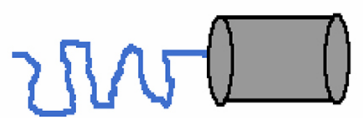

\section{Copolímero Coil-Coil}

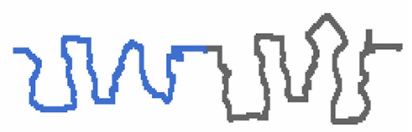

Figura 2. Copolímeros anfifílicos coil-coil e rod-coil. Blocos flexíveis representados em linha e bloco rígido representado em cilindro. Blocos hidrofílicos representados em azul e blocos hidrofóbicos representados em cinza/preto

\section{MECANISMOS DE FORMAÇÃO DOS POLIMEROSSOMOS}

Os estudos envolvendo o processo de formação dos PL são recentes. Em 1994 sugeriu-se a formação de estruturas vesiculares poliméricas e em 1995 foi descrita a formação de vesículas poliméricas a partir de CA. ${ }^{11,41}$ Desde 1999, Dennis Discher tem um sido um dos principais cientistas a estudar os PL. Além de ter descrito pela primeira vez as vesículas com tal nomenclatura, Discher também relatou diversas informações sobre organização conformacional, produção e aplicação destas nanoestruturas.

Dois mecanismos têm sido citados para explicar a formação dos
PL: um baseado no crescimento de micelas em bicamadas que se auto-organizam em vesículas e outro também embasado no crescimento de micelas, entretanto, acrescentando o efeito de difusão de solvente para o interior das micelas e posteriormente a formação das vesículas. ${ }^{42}$ Desse modo, tem sido aceito que as micelas esféricas, que são núcleos esféricos rodeados por uma corona polimérica, seriam os auto-agregados dos quais se formariam os demais tipos. ${ }^{35} \mathrm{~A}$ partir de 2005, um grupo liderado por Giuseppe Battaglia vem realizando amplos estudos na temática, nos quais descreve que CA em concentrações mais elevadas se auto-organizam em cristais líquidos liotrópicos e a transição da fase liotrópica para estruturas isotrópicas ocorre, por exemplo, com a passagem das lamelas em pilhas para estruturas fechadas (vesículas) com aumento da fase aquosa. ${ }^{43}$

Durante um período de tempo, o fenômeno de auto-agregação de moléculas anfifílicas foi ponto de discórdia entre cientistas da área físico-química, que aceitavam que forças físicas e a minimização de energia livre no sistema levavam à agregação, e aqueles da área biológica, que consideravam que a geometria das moléculas resultaria no processo de auto-agregação. ${ }^{44}$ Atualmente, aceita-se que esse conjunto de proposições se completam e explicam a auto-agregação. Como já mencionado, copolímeros anfifílicos se auto-agregam em meio aquoso dependendo da relação entre as massas moleculares dos blocos hidrofílico e hidrofóbico e da concentração de agregação crítica (CAC). Esse processo ocorre em razão do efeito hidrofóbico que demanda minimização da energia livre das moléculas de água próximo à superfícies não polares bem como da solvatação das "cabeças hidrofílicas" do copolímero (forças de hidratação), podendo resultar em diversas morfologias. A quantidade crítica de água para o início da agregação depende dos seguintes fatores: (1) concentração de polímero, (2) temperatura, (3) composição de copolímero (estrutura química, proporção e massa molecular de cada bloco de homopolímero) e (5) propriedades do solvente..$^{42}$

A morfofologia resultante da auto agregação desses polímeros pode ser deduzida a partir do parâmetro crítico de empacotamento ou critical packing parameter ( $p$ ) descrito na equação 1. Este conceito foi introduzido para moléculas anfifílicas em 1975 por Israellachvilli. ${ }^{45}$

$$
p=\frac{v}{a_{0}+l_{c}}
$$

em que: v é o volume da cadeia hidrofóbica, $a_{\mathrm{o}}$ é a área da seção transversal da cabeça polar do copolímero e $l_{\mathrm{c}}$ é o comprimento da cadeia hidrofóbica. É importante ressaltar que na época de divulgação deste conceito não havia conotações para nanoestruturas do tipo PL. ${ }^{44}$

A Tabela 1 traz a classificação teórica dos sistemas formados de acordo com o valor de $p$.

Tabela 1. Classificação teórica dos sistemas formados a partir do critical packing parameter $(p)$

\begin{tabular}{cc}
\hline Critical packing parameter & Estruturas formadas \\
\hline$p<1 / 3$ & Micelas esféricas \\
$\mathbf{1} / \mathbf{3}<p<1 / 2$ & Micelas cilíndricas \\
$\mathbf{1} / \mathbf{2}<p<1$ & Lamelas flexíveis e vesículas poliméricas \\
$p=1$ & Lamelas planares \\
\hline
\end{tabular}

Para copolímeros com porção hidrofílica formada por PEG, pode-se ainda inferir o tipo de agregado formado a partir do parâmetro $f(0<f<1)$, que representa a fração de volume hidrofílico do copolímero e o qual pode ser convertido em porcentagem, a partir dos valores de massa das frações, usando o valor da densidade dos homopolímeros (Tabela 2). ${ }^{45}$ A Figura 3 apresenta uma ilustração 
destes dois parâmetros que interferem na geometria das nanoestruturas e estão intimamente ligados.

Tabela 2. Classificação teórica dos sistemas formados a partir do valor da fração do volume hidrofílico $(f)$

\begin{tabular}{cc}
\hline Fração hidrofílica $(f) \%$ & Estruturas poliméricas formadas \\
\hline $\mathbf{4 0}<f<\mathbf{2 5}$ & Vesículas \\
$\mathbf{5 0}<f<\mathbf{4 0}$ & Micelas cilíndricas \\
$f>\mathbf{5 0}$ & Micelas esféricas \\
\hline
\end{tabular}

Do ponto de vista termodinâmico, a formação das micelas e vesículas é determinada pela energia livre do sistema, expressa pela equação 2:

$$
\Delta G=\Delta H-T \Delta S
$$

em que $\Delta \mathrm{G}, \Delta \mathrm{H}$ e $\Delta \mathrm{S}$ são, respectivamente, as variações de energia livre de Gibbs, entalpia e entropia no processo de auto-agregação; T é a temperatura.

A contribuição entálpica advém do balanço das interações intra e inter moleculares entre o solvente e as moléculas de copolímero, incluindo interações de van der Waals e ligações de hidrogênio. O ganho entrópico advém da liberação das moléculas de água inicialmente estruturadas na vizinhança dos grupos hidrofóbicos do copolímero. ${ }^{46}$ Neste sentido, o parâmetro de segregação $\chi N$ expressa o grau de segregação entre os segmentos hidrofílicos(a) e hidrofóbicos(b) do copolímero, $\chi$ refere-se a interação entre os diferentes blocos denominada de interação Flory-Huggins e $N$ ao grau de polimerização $\left(\mathrm{N}=\mathrm{N}_{\mathrm{a}}+\mathrm{N}_{\mathrm{b}}\right)$. Um limite amplo de $\chi N$ resulta em energia interfacial maior e menor perda da entropia, consequentemente, os segmentos estão altamente segregados. Na presença de um solvente seletivo para um dos blocos, a separação dos CA em uma microfase deve considerar também a interação de cada um dos seguimentos com tal solvente. ${ }^{42}$

\section{MÉTODOS DE PREPARO}

Os principais métodos de preparo de PL incluem auto-agregação por simples dissolução, hidratação de um filme polimérico fino, troca de solvente ou co-solvente (solvent-switching) com precipitação do copolímero e dupla emulsificação com evaporação do solvente. ${ }^{47,48} \mathrm{~A}$ formação espontânea de PL é relatada por alguns autores em certas condições, por exemplo, alguns copolímeros são solúveis em determinadas faixas de $\mathrm{pH} .{ }^{49}$ No entanto, no geral são necessárias etapas que forneçam energia ao sistema. ${ }^{50}$

O método da hidratação do filme consiste na hidratação sob agitação vigorosa de um filme polimérico fino formado a partir da lenta evaporação de um solvente orgânico no qual o copolímero está dissolvido. ${ }^{51}$ Pode-se, ainda, empregar outras técnicas como agitação por ultrassom (sonicação), ciclo de resfriamento e aquecimento ou extrusão, visando reduzir o tamanho das vesículas e diminuir a polidispersão. $^{52}$

A sonicação é feita em banho de ultrassom e a extrusão com os mesmos extrusores utilizados para LPs, com passagens sequenciais por membranas de policarbonato com poros de tamanho decrescente. A sonicação gera energia por meio do fenômeno da cavitação, que consiste na expansão e contração de bolhas de gás em um líquido exposto a ondas acústicas de pressão com (cavitação com colapso) ou sem (cavitação estável) ruptura das bolhas. A modificação de tamanho de vesículas poliméricas pode ser explicada pelo mesmo fenômeno descrito para vesículas lipídicas, o qual se sustenta no fato de que a oscilação das bolhas cria uma pressão local resultando na quebra das vesículas maiores em fragmentos que se reorganizam em vesículas menores. ${ }^{53} \mathrm{~A}$

\section{Copolímero anfifilicos}

\section{$2 \pi 2505$}

Copolímero dibloco

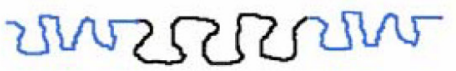

Copolímero tribloco
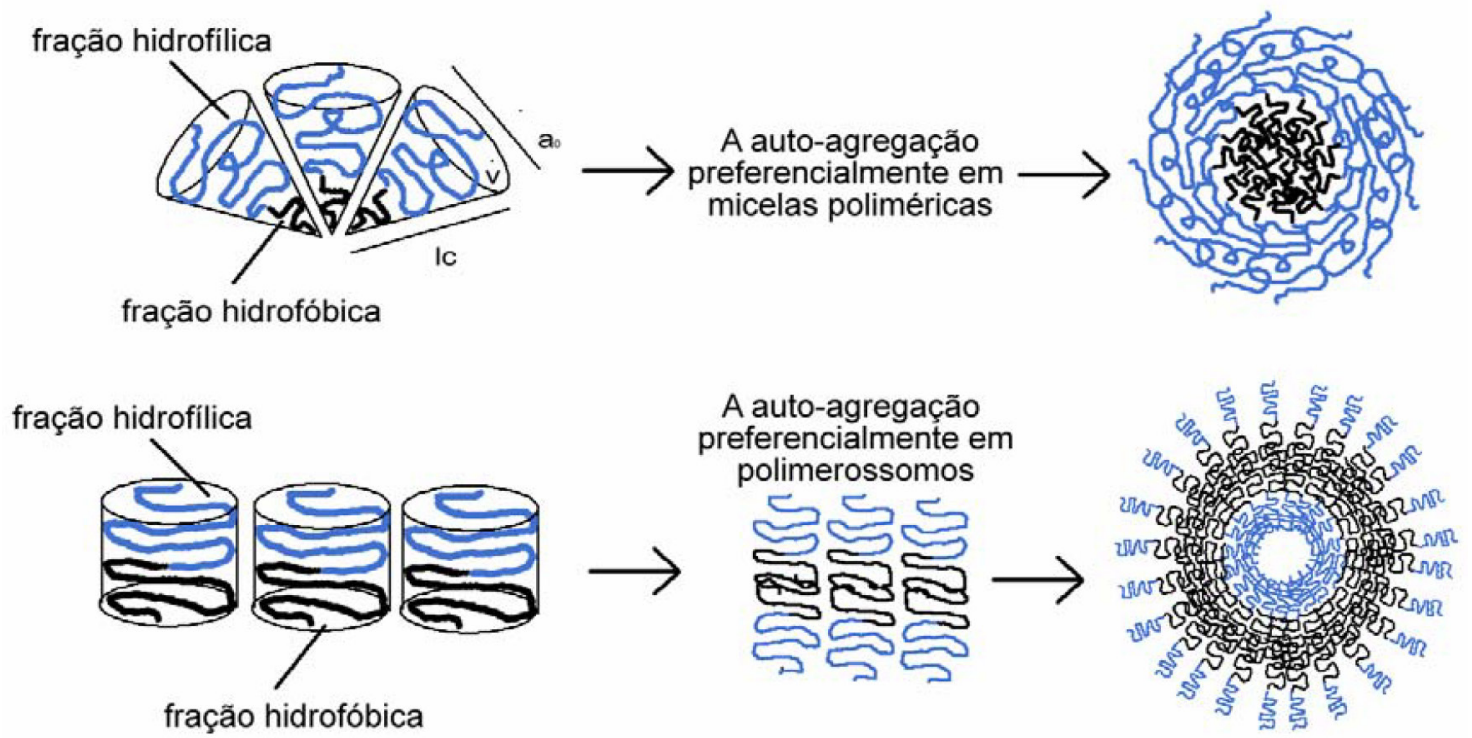

Figura 3. Representação esquemática da organização geométrica de PL para um copolímero dibloco. De acordo com os tamanhos e proporções dos blocos hidrofílico e hidrofóbico, expressos matematicamente pela fração hidrofilica (f) ou pelo critical packing parameter (p), ocorre a agregação preferencialmente em micelas ou vesículas poliméricas (polimerossomos) 
extrusão também leva à diminuição de tamanho por promover a ruptura das vesículas quando forçadas através da membrana com tamanho de poro definido e reorganização em PL com tamanho menor.

Liu e colaboradore ${ }^{54}$ descreveram PL biodegradáveis formados a partir de copolímero tribloco de polietilenoglicol- $\beta$-policaprolactona$\beta$-poli(2-dimetilaminoetilmetacrilato), obtidos pelo método de hidratação do filme. O copolímero foi dissolvido em clorofórmio e a remoção do solvente foi feita lentamente com aplicação de vácuo por três a seis horas. A hidratação do filme foi feita com solução tampão contendo proteínas, seguida de sonicação por três minutos e agitação por 12 horas a $60{ }^{\circ} \mathrm{C}$. De modo semelhante ao protocolo anterior, Ahmed e Discher ${ }^{45}$ elaboraram PL empregando copolímero de polietilenoglicol-poli(ácido láctico) (PEG-PLA) e polietilenoglicol-policaprolactona (PEG-PCL) para encapsular doxorrubicina. $\mathrm{O}$ método resultou em formação das vesículas com alto grau de polidispersão, uma vez que não foram realizadas etapas de sonicação e extrusão. Algumas variáveis devem ser consideradas para aplicação deste método: volume da solução de hidratação, temperatura durante o processo de hidratação, velocidade e tempo de agitação. É relatado que hidratação sem agitação aumenta a polidispersão, resultando em ampla distribuição de tamanho das vesículas. A hidratação sob agitação vigorosa, por sua vez, reduz o tamanho das vesículas formadas. ${ }^{55}$ Apesar de amplamente descrito na literatura, esse método apresenta desvantagens como a tendência em formar sistemas polidispersos, a utilização de longos períodos de tempo durante a hidratação para obtenção das vesículas, bem como a necessidade de métodos para diminuição do tamanho das vesículas, como por exemplo, extrusão da amostra final..$^{56}$

O método da troca do solvente consiste em inicialmente dissolver os CA em um solvente orgânico e, em seguida, adicionar lentamente uma fase aquosa (ou injetar a fase orgânica em uma fase aquosa), levando à precipitação das vesículas. Por isso, pode ser denominado também de nanoprecipitação. Posteriormente é realizada e remoção do solvente por técnicas de diálise ou evaporação. ${ }^{57}$ Este método foi empregado por Lee e colaboradores ${ }^{58}$ para elaboração de PL de PEG-PLA, o copolímero foi dissolvido em clorofórmio e adicionado em água com posterior agitação em vórtex. Em seguida, o solvente foi removido por agitação magnética. Marsden e colaboradores ${ }^{59}$ descreveram o preparo de PL seguindo a mesma técnica para diferentes copolímeros e empregando tetrahidrofurano (THF) como solvente. Neste trabalho, a evaporação do solvente ocorreu sob vácuo em evaporador rotativo.

A técnica da dupla emulsificação é descrita como uma possibilidade para obtenção de PL menos polidispersos. Nesse método, são formadas emulsões do tipo água-em-óleo-em-água, sendo que os copolímeros dissolvidos na fase oleosa se auto-organizam em PL após evaporação da fase orgânica. ${ }^{60} \mathrm{Na}$ obtenção de PL por dupla emulsificação podem ser usados dispositivos microfluídicos, os quais permitem a manipulação precisa de microfluxos em microcanais para gerar gotículas de emulsão uniformes com controle de tamanho das estruturas. ${ }^{61,62}$

Um método alternativo, a eletroporação, vem sendo utilizado para a encapsulação de biomoléculas em PL pré-formados. Esta consiste na aplicação de um campo elétrico que abre temporariamente poros nanométricos na membrana da vesícula permitindo a passagem das moléculas que se deseja encapsular. A abertura dos poros depende do tamanho das vesículas, voltagem aplicada, número, duração e frequência dos pulsos. ${ }^{63}$ Alguns trabalhos relatam sucesso no emprego desta técnica para encapsulação de biomoléculas como proteínas. ${ }^{64}$

\section{PRINCIPAIS TÉCNICAS DE CARACTERIZAÇÃO}

Uma extensa revisão recentemente publicada elencou dezessete técnicas para caracterização de PL em meio aquoso, com intuito de descrever tamanho, lamelaridade, propriedades elásticas, carga, espessura da bicamada e polaridade. $\mathrm{O}$ mesmo trabalho divide as técnicas em cinco grupos: técnicas de espalhamento, métodos visuais, manipulação física e eletromagnética, ensaios de purificação e ferramentas de simulação. ${ }^{65}$

O tamanho fornecido pela técnica de espalhamento de luz dinâmico (do inglês dynamic light scattering, $D L S$ ) baseia-se no espalhamento da luz incidente a partir de um laser a uma intensidade induzida pelo movimento Browniano das partículas em solução, fornecendo o raio ou diâmetro hidrodinâmico a partir da equação de Stokes-Einstein, e assim deve ser correlacionado com análises microscópicas. ${ }^{66}$ Resultados de DLS podem sugerir agregação dos sistemas ou indicar mudanças nos tamanhos dos sistemas mediante alterações de condições experimentais. ${ }^{67,68}$

$$
D_{f}=\frac{\mathrm{k}_{B} \mathrm{~T}}{6 \pi \eta \mathrm{R}_{\mathrm{h}}}
$$

em que: $\mathrm{D}_{\mathrm{f}}$ é o coeficiente de difusão, $\mathrm{k}_{B}$ é a constant de Boltzmann $\left(1,38064852 \times 10^{-23} \mathrm{~J} / \mathrm{K}\right), \mathrm{T}$ a temperatura, $\eta$ a viscosidade do solvente e $\mathrm{R}_{\mathrm{h}}$ é o raio hidrodinâmico.

A microscopia eletrônica de varredura (MEV), a microscopia eletrônica de transmissão (MET) e a criomicroscopia de transmissão (Crio-MET) são técnicas amplamente empregadas para visualização dos PL, sendo que a primeira permite observar a forma e as outras duas são mais precisas no que diz respeito à demonstração dos tipos de estruturas formadas permitindo avaliar a bicamada polimérica. ${ }^{57,66} \mathrm{Já}$ a microscopia confocal (MCF), uma vez que se trata de uma técnica de microscopia óptica, requer a produção de vesículas grandes (micrométricas), do inglês, giant vesicles (sem técnicas de extrusão e sonicação). Nessa técnica são empregados marcadores fluorescentes como nile red, isotiocianato de fluoresceína (acumulam-se na membrana) e rodamina-B (acumula-se no interior aquoso) para visualização das vesículas. ${ }^{69,70}$ A microscopia de fluorescência também necessita de marcador fluorescente nas vesículas e permite estudar processos dinâmicos como difusão. ${ }^{66}$ A microscopia de força atômica (MFA) pode ser usada para avaliar características mecânicas da bicamada dos $\mathrm{PL}^{71}$ como fizeram Chen e coloaboradores que, por meio de MFA, avaliaram a rigidez de membranas de poliestireno-b-poli(ácido)acrílico. ${ }^{72}$

Assim como para outros NC, a taxa de encapsulação pode ser calculada diretamente nos sistemas após tratamentos que desagregam os PL ou indiretamente no sobrenadante após centrifugação dessas nanoestruturas. No primeiro caso são empregados tensoativos ou solventes orgânicos que permitem a dissolução do polímero e, assim, liberação do fármaco encapsulado para quantificação direta. ${ }^{73}$ Já no segundo caso, quantifica-se o fármaco não-encapsulado no sobrenadante e, por diferença, estima-se a porcentagem que foi encapsulada.

A análise do potencial zeta (PZ) na maioria das vezes é comparativa para avaliação dos sistemas após encapsulação do fármaco. ${ }^{74}$ Como o valor de potencial zeta está relacionado com a carga do polímero, PL formados por copolímeros anfifílicos contendo polióxido de etileno como porção polar apresentam valores de potencial zeta próximos a zero e a estabilização dos PL ocorre por efeito estérico.

Outra técnica que pode ser aplicada é o espalhamento de raio-X a baixo ângulo (do inglês, Small angle X-ray scattering, SAXS), técnica em que o espalhamento de raios-X é realizado em uma estreita faixa de ângulo $\left(0,1-10^{\circ}\right)$ que fornece informações quanto à espessura e estrutura interna das vesículas. ${ }^{75}$

\section{APLICAÇÕES E INOVAÇÕES}

As aplicações dos PL em geral são semelhantes às já conhecidas para os lipossomos. ${ }^{76} \mathrm{~A}$ Tabela 3 lista algumas aplicações de diferentes sistemas de PL. Como já citado antes, a estrutura polimérica faz com 
que apresentem propriedades que os tornam menos susceptíveis a rápida degradação no organismo e menos imunogênicos.

Inovações na estrutura dos PL refletem uma evolução na ideia original destes NC como sistemas de armazenamento estável de um fármaco em razão da natureza polimérica da membrana e abrem vertentes para conceituá-los também como "sistemas inteligentes" de liberação de fármacos, especialmente em razão das dificuldades já relatadas para liberação. ${ }^{89}$ Assim, auto-agregados supramoleculares podem responder a estímulos externos (por exemplo, $\mathrm{pH}$, temperatura, campos magnéticos, luz, processos redox). ${ }^{90}$ Na Tabela 4 são apresentadas as modificações mais comuns em PL de maneira a funcionalizá-los. Outra modificação pode ser na morfologia das vesículas, uma vez que a forma final dos PL é resultado de forças que controlam a membrana. Um exemplo de modificação morfológica refere-se ao desenvolvimento de sistemas tubulares com morfologia cilíndrica alongada, os quais penetrariam mais facilmente no ambiente intracelular. Para isto, fosfolipídeos e colesterol poderiam ser incorporados na composição dos PL. ${ }^{51}$

Tabela 3. Aplicações para polimerossomos obtidos a partir de diferentes copolímeros e técnicas

\begin{tabular}{cccc}
\hline Copolímero & Técnica & Aplicação & Caracterização das vesículas \\
\hline $\begin{array}{c}\text { Poli(dimetilsiloxano)-b-poli(2-metil-2- } \\
\text { oxazolona) }\end{array}$ & $\begin{array}{c}\text { Hidratação do filme e } \\
\text { extrusão }\end{array}$ & $\begin{array}{c}\text { Permeação da barreira hematoen- } \\
\text { cefálica por vesículas poliméri- } \\
\text { cas conjugadas com anticorpos }\end{array}$ & MEV e DLS \\
\hline
\end{tabular}

Poli(óxido de etileno)-poli(2-

$\begin{array}{cc}\text { dimetilamino metacrilato de etila) } & \text { Formação espontânea com } \\ \text { modificado com presença do } & \text { ajuste de pH para } 7,8 \mathrm{com} \\ \text { grupamento cisteamina que torna o } & \text { posterior agitação a } 37^{\circ} \mathrm{C} \\ \text { bloco solúvel em meio fisiológico mas } & \text { para formação de ligações } \\ \text { capaz de formar polimerossomos } & \text { dissulfídicas } \\ \text { acima do pH 7,8 } & \end{array}$

Liberação intracelular de pro- $\quad$ MCF, DLS. Além de PZ para teínas determinação da carga

Poli(óxido de etileno)-bpoli(carbonato de trimetileno ) Formação espontânea

Liberação intracelular de proteínas

MET e MCF

Monometoxi-poli(óxido de etileno)-b-poli(\&-caprolactona) e Poli(etilenoglicol)maleimide

Nanoprecipitação

Permeação da transferrina pela barreira hematoencefálica

Crio-MEV, DLS e potencial zeta

Poli(óxido de etileno)-b-poli(\&-caprolactona)-b-poli(2-dietilaminoetil metacrilato)
Hidratação do filme com sonicação

Troca de solvente seguida de sonicação

Nanoprecipitação

Liberação intracelular da doxurrubicina
Liberação de gencitabima e

doxurribicina em células cancerígenas teínas láctico) (Neste caso o polímero

foi conjugado com cloridrato de cistamina)
Poli(trimetileno-carbonato)-b-poli( $\mathrm{L}$ ácido glutâmico)

Liberação intracelular de pro-

DLS e MCF

ácido glutâmico)

Poli(óxido de etileno)-b-poli(lisina)-b-

Encapsulação de fármaco

anticancerígeno hidrofóbico

\begin{tabular}{cc}
\hline $\begin{array}{c}\text { Poli(2-metacriloiloxi(etilfosforilco- } \\
\text { lina)-co-poli(2-(diisopropilamino) } \\
\text { etilmetacrilato }\end{array}$ & Hidratação do filme \\
\hline $\begin{array}{c}\text { Poli(óxido de etileno)-poli-(D,L-ácido } \\
\text { láctico-co-glicólico) }\end{array}$ & Nanoprecipitação \\
\hline $\begin{array}{c}\text { Glicopolipetídeo-b-Poli(óxido de } \\
\text { etileno) }\end{array}$ & Nanoprecipitação \\
\hline $\begin{array}{c}\text { Poli(óxido de etileno)-b- } \\
\text { poli(caprolactona) }\end{array}$ & $\begin{array}{c}\text { Hidratação do filme e troca } \\
\text { de solvente }\end{array}$
\end{tabular}

DLS, MET e MFA

oxazolina)
Poli-(2-metil oxazolina)-

DLS e PZ policaprolactona

Nanoprecipitação

(camptocina) e hidrofílico (doxurrubicina) no mesmo sistema

de liberação intracelular

Liberação intracelular da doxor-

rubicina e nuclear em células do

tipo melanoma

cefálica da lactoferrina

Encapsulação simultânea de modelos de fármacos hidrofílico e hidrofóbico

Liberação direcionada para célu-

las tumorais com reconhecimento de biomarcadores por anticorpos

Poli-(2-metil oxazolina)poli(dimetilsiloxano)-poli(2-metil

Reidratação do filme

Nanorreator para encapsulação de proteína poli(dimetilsiloxano)-poli(2-metil oxazolina)

Dissolução direta

Desenvolvimento de uma organela artificial que mimetize um peroxissomo com encapsulação de duas enzimas

Aplicação teranóstica na obtenção de imagens em ressonância magnética nuclear

DLS e MET

Poli (carbonato de trimetileno)-bpoli(ácido L-glutâmico)

Nanoprecipitação

DLS e MET

82

Crio-MET e PZ

83

DLS, SLS, MFA,

Crio-MEV e MET

Crio-MET, DLS e PZ

85

85

DLS e MET

86

12


Tabela 4. Modificações que podem ser aplicadas aos polimerossomos para funcionalizá-los

\begin{tabular}{lll}
\hline Tipo & \multicolumn{1}{c}{ Modificação } & \multicolumn{1}{c}{ Aplicação } \\
\hline Bioredútivel & $\begin{array}{l}\text { Introdução de agentes crosslinking contendo pontes dis- } \\
\text { sulfeto, as quais são reduzidas na presença de glutationa } \\
\text { que está presente em excesso no interior celular em } \\
\text { relação ao ambiente externo. }\end{array}$ & Liberação intracelular de fármacos \\
\hline pH-Responsivos & Introdução de ligantes sensíveis ao pH & $\begin{array}{l}\text { Liberação em regiões com baixo pH como tumores } \\
\text { tecidos inflamados, lisossomos }\end{array}$ \\
\hline Responsivos a enzimas & $\begin{array}{l}\text { Substratos responsivos a enzimas são ligados covalente- } \\
\text { mente aos polímeros que formarão os polimerossomos }\end{array}$ & $\begin{array}{l}\text { Liberação na presença de enzimas liberadas, por exem- } \\
\text { plo, no processo inflamatório ou por tecidos tumorais }\end{array}$ \\
\hline Fotossensíveis & Grupamentos cromóforos são ligados ao copolímero & Terapia fotodinâmica \\
\hline Termorresponsivos & $\begin{array}{l}\text { Polímeros com transição de fases em temperaturas } \\
\text { desejadas }\end{array}$ & $\begin{array}{l}\text { Liberação em células tumorais e tecidos inflamados, } \\
\text { os quais que apresentam diferença de temperatura em } \\
\text { relação à células/tecidos saudáveis }\end{array}$ \\
\hline Alvo-específicos & $\begin{array}{l}\text { Ancoração de grupamentos que reconhecerão o alvo, } \\
\text { como aptâmeros por exemplo }\end{array}$ & Liberação sítio-dirigida com captação celular \\
\hline
\end{tabular}

\section{CONSIDERAÇÕES FINAIS}

Desde que o conceito de "bala mágica" de Paul Ehrlich foi extrapolado para drug delivery com os lipossomos, descritos inicialmente nos anos 60, podemos dizer que esta ideia não parou de evoluir, especialmente no campo da nanobiotecnologia. Atualmente, o conceito pode ser aplicado aos polimerossomos, nanoestruturas que reúnem todas as vantagens do sistema vesicular com núcleo aquoso e bicamada de caráter apolar característico dos lipossomos, mas apresentam vantagens de nanopartículas poliméricas, especialmente no que diz respeito à robustez. Desde a publicação inicial do termo polimerossomo e sua descrição como nanocarreador no final dos anos 90 até hoje, o próprio conceito de auto-organização ou self-assembly de compostos anfifílicos, que é a base teórica da formação destas vesículas, vem sendo expandido. Novos estudos vêm sendo realizados em relação à elaboração de polimerossomos, com foco nas características dos copolímeros como sensibilidade a certas faixas de temperatura e/ou $\mathrm{pH}$ que podem determinar a auto-agregação e variações nas técnicas de produção que permitem otimizar este processo. A variedade de copolímeros com diferentes características, capazes de direcionar fármacos para alvos específicos e controlar a velocidade de liberação e degradação, o aumento da meia vida dos fármacos encapsulados em razão da furtividade típica de certos materiais poliméricos, a possibilidade de terapia combinada com fármacos hidrofílicos no interior aquoso e fármacos hidrofóbicos na membrana, além de todas as outras vantagens de transportadores em escala nanométrica, tornam os polimerossomos uma abordagem atual e inteligente para drug delivery.

\section{AGRADECIMENTOS}

Os autores agradecem ao CNPq, à CAPES e à FAPESP (processo 2013/08617-7 - Projeto temático e 2014/10456-4 - bolsa de doutorado para A. C. Apolinário).

\section{REFERÊNCIAS}

1. Dimer, F. A.; Friedrich, R. B.; Beck, R. C. R.; Pohlmann, A. R.; Quim Nova. 2015, 36, 1520.

2. Pachioni-Vasconcelos, J. D. A.; Lopes, A. M.; Apolinário, A. C.; Valenzuela-Oses, J. K.; Costa, J. S.R.; Nascimento, L. O.; Pessoa Jr., A.; Barbosa, L. R. S.; Rangel-Yagui, C. O.; Biomater Sci. 2016, 4, 205.

3. Wicki, A.; Witzigmann, D.; Balasubramanian, V.; Huwyler, J.; J. Controlled Release 2015, 200, 138.
4. Safari, J.; Zarnegar, Z.; J. Saudi Chem. Soc. 2014, 18, 85.

5. Alcalá-Alcalá, S.; Benítez-Cardoza, C. G.; Lima-Muñoz, E. J.; PiñónSegundo, E.; Quintanar-Guerrero, D.; Int. J. Pharm. 2015, 489, 139.

6. Cheng, X.; Lee, R. J.; Adv. Drug Delivery Rev. 2016, 99, 129.

7. Ray, C.; Bhattacharya, D.; Sahu, S. K.; Dalton Trans. 2016, 45, 72963.

8. Li, X.; Anton, N.; Arpagaus, C.; Belleteix, F.; Vandamme, T. F.; J. Controlled Release 2010, 147, 304.

9. Otsuka, H.; Nagasaki, Y.; Kataoka, K.; Adv. Drug Delivery Rev. 2012, 64, 246.

10. Kunitake T, Nakashima N, Takarabe K, Nagai M, Tsuge A, Yanagi H.; J. Am. Chem. Soc. 1981, 28, 5945.

11. Zhang, L.; Eisenberg, A.; Science 1995, 268, 5218.

12. Discher, B. M.; Won, Y. Y; Ege, D. S.; Lee, J. C.; Bates, F. S.; Discher, D. E.; Hammer, D. A.; Science 1999, 284, 1143.

13. Bangham, D.; Standish, M. M.; Watkins, J. C.; J. Mol. Biol. 1965, 13, 238.

14. Santos, N. C.; Castanho, M. A. R. B.; Quim. Nova. 2002, 25, 1181.

15. Pasut, G.; Paolino, D.; Celia, C.; Mero, A.; Joseph, A. S.; Wolframd, J.; Cosco, D.; Schiavon. O.; Shen, H.; Fresta, M.; J. Controlled Release 2015, 199, 106.

16. Brinkhuis, R. P.; Rutjes, F. P. J. T.; van Hest, J. C. M.; Polym. Chem. 2011, 2, 1449.

17. Schaffazick, S. R.; Guterres, S. S.; Lucca F. L.; Pohlmann, A. R.; Quim Nova 2003, 26, 726.

18. Oliveira, A.G.; Scarpa, M. V.; Correa, M. A.; Rodrigues, C. L. F, Formariz, T. P.; Quim Nova 2004, 27, 131.

19. Durán, N.; Durán, M.; Tasic, L.; Marcato, P. D.; Quim Nova 2010, 33, 151.

20. Yoshizaki, Y.; Yuba, E.; Sakaguchi, N.; Koiwai, K.; Harada, A.; Kono, K.; Biomaterials 2014, 35, 8186.

21. Kim, S. J.; Kwon, S. S.; Jeon, S. H.; Yu, E. R; Park, S. N.; Int. J. Cosmet. Sci. 2014, 36, 553.

22. Messager, L.; Gaitzsch, J.; Chierico, L.; Battaglia, G.; Curr. Opin. Pharmacol. 2014, 18, 104.

23. Mosca, M.; Ceglie, A.; Ambrosone, L.; Chem. Phys. Lipids 2011, 164, 158.

24. Toh, M-R.; Chiu, G. N. C.; Asian J. Pharm. Sci. 2013, 8, 88.

25. Lasic, D. D.; Trends Biotechnol. 1998, 16, 307.

26. Maherani, B.; Arab-Tehrany, E.; Mozafari, M. R., Gaiani, C.; Linder, M.; Curr. Nanosci. 2011, 7, 436.

27. Onaca, O.; Enea, R.; Hughes, D. W.; Meier, W.; Macromol. Biosci. 2009, 9, 129.

28. Schacher, F. H.; Rupar, P.; Manners, I.; Angew. Chem. 2012, 51, 7898.

29. Chang, H.; Sheng, Y.; Tsao, H.; Soft Matter 2014,10,6373. 
30. Zhang, X.; Qi, J.; Lu, Y.; He, W.; Li, X.; Wu, W.; Nanomedicine 2014, $10,167$.

31. Le Meins, J-F.; Sandre, O.; Lecommandoux, S.; EPJSFH 2011, 34, 1.

32. Discher, D. E.; Ortiz, V.; Srinivas, G.;. Kleinb. M. L.; Kim, Y.; Christian, D.; Cai, S.; Photos, P.; Ahmed, F.; Prog. Polym. Sci. 2007, 32, 838.

33. Pawar, P. V.; Gohil, S. V.; Jain, J. P.; Kumar, N.; Polym. Chem. 2013, 4, 160.

34. Discher, D. E.; Eisenberg, A.; Science 2002, 297, 967.

35. Mai, Y.; Eisenberg, A.; Chem. Soc. Rev. 2012, 41, 5969.

36. Discher, D. E.; Ahmed, F.; Annu. Rev. Biomed. Eng. 2006, 8, 323.

37. Gentekos, D. T.; Dupuis, L. N.; Fors, B. P.; J. Am. Chem. Soc. 2016, 138, 1848.

38. Lin, Y.; Chang, H-Y.; Sheng, Y-J.; Tsao, H-K.; Soft Matter 2013, 9, 4802.

39. Lee, M.; Cho, B-K.; Zin, W-C.; Chem. Rev. 2001, 101, 3869.

40. Olsen, B. D.; Segalman, R.; Mater. Sci. Eng. 2008, 62, 37.

41. Honda, C.; Sakaki, K.; Nose, T.; Polymer 1994, 35, 5309.

42. Bleul, R.; Thiermann, R.; Maskos, M.; Macromolecules 2015, 48, 7396.

43. Battaglia, G.; Ryan, A. J.; Nat. Mater. 2005, 4, 869.

44. Israelachvili, J. N.; Mitchell, D. J.; Ninham, W. B.;. J. Chem. Soc., Faraday Trans. 1975, 72, 1525.

45. Ahmed, F.; Discher, D. E.; J. Controlled Release. 2004, 96, 37.

46. Hocine, S.; Li, M-H.; Soft Matter 2013, 9, 5839.

47. Liao, J., Wang, C.; Wang, Y.; Luo, F.; Qian. Z.; Curr. Pharm. Des. 2012 18,3432 .

48. Shen, H.; Eisenberg, A.; J. Phys. Chem. B. 1999, 103, 9473.

49. Sun, H.; Meng, F.; Cheng, R.; Deng, C.; Zhong, Z.; Acta Biomater. 2014, $10,2159$.

50. Howse, J. R.; Jones, R. L.; Battaglia, G.; Ducker, R. E., Leggett, G. J.; Ryan, A. J.; Nat. Mater. 2009, 8, 507.

51. Robertson, J. D.; Yealland, G.; Avila-Olias, M.; Chierico, L.; Bandmann, O.; Renshaw S. A.; Giuseppe B.; ACS Nano 2014, 8, 4650.

52. Letchford, K.; Burt, H.; Eur. J. Pharm. Biopharm. 2007, 65, 259.

53. Richardson, E. S.; Pitt, W. G.; Woodbury, D. J.; Biophys J. 2007, 93, 4100.

54. Liu, G.; Ma, S.; Li, S.; Cheng, R.; Meng, F.; Liu, Haiyan, L.; Zhong, Z.; Biomaterials 2010, 31, 7575.

55. Photos, P. J.; Bacakova, L.; Discher, B.; Bates, F. S.; Discher, D. E.; J. Controlled Release 2003, 90, 323.

56. O’Neil, C. P.; Suzuki T.; Demurtas, D.; Finka, A.; Hubbell, J.; Langmuir 2009, 25, 9025.

57. Lee, J. S.; Feijen, J.; J. Controlled Release 2012, 161, 473.

58. Lee, Y.; Chang, J-B.; Kim, H. K.; Park, T. G.; Macromol. Res. 2006, 14, 359.

59. Marsden, H. R.; Gabrielli, L.; Kros, A.; Polym. Chem. 2010, 1,1512.

60. Shum, H. C.; Zhao, Y. J.; Kim, S. H.; Weitz, D.; Angew. Chem. 2011, 50, 1648.

61. Jang, W-S.; Park, S. C.; Kim, M.; Doh, J.; Lee, D.; Hammer, D.; Macromol. Rapid Commun. 2015, 36, 378.

62. Zhang, M.; Wang, W.; Xie, R.; Ju, X.; Liu, Z.; Jiang, L.; Chen, Q.; Chu, L.; Particuology 2015, 1, 14.

63. Bain, J.; Ruiz-Pérez, L.; Kennerley, A. J.; Muench, S. P.; Thompson, R.; Giuseppe B.; Staniland, S. S.; Sci. Rep. 2015, 5, 14311.

64. Wang, L.; Chierico, L.; Little, D.; Patikarnmonthon, N.; Yang, Z.; Azzouz, M.; Madsen, J.; Armes, S. P.; Battaglia, G.; Angew. Chem. 2012, 51, 11122 .

65. Habel. J.; Ogbonna, A.; Larsen, N.; Cherré S.; Kynde, S.; Roi, S.; Koji, M.; Kinoshita, S.; Grethe, K.; Jensen, V.; Hansen, J. S.; Almdal, K.; Hèlix-Nielsen, C.; RSC Adv. 2015, 5, 79924.
66. Kita-Tokarczyk, K.; Grumelard, J.; Haefele, T.; Meier, W.; Polymer 2005, 46, 3540 .

67. Smart, T. P.; Fernyhough, C.; Ryan, A. J.; Battaglia, G.; Macromol. Rapid Commun. 2008, 29, 1855.

68. Du, Y.; Chen W.; Zheng, M.; Meng, F.; Zhong, Z.; Biomaterials 2012, $29,7291$.

69. Liu, G.; Ma, S.; Li, S.; Liu, G.; Ma, S.; Li, S.; Cheng, R.; Meng, F.; Liu, H.; Zhong, Z.; Biomaterials 2010, 31, 7575.

70. Gaitzsch, J.; Appelhans, D.; Gräfe, D.; Schwille, P.; Voit, B.; Chem. Commun. (Cambridge, U. K.) 2011, 47, 3466.

71. Jaskiewicz, K.; Makowski, M.; Kappl, M.; Landfester, K.; Kroeger, A.; Langmuir 2012, 28, 12629.

72. Chen, Q.; Schönherr, H.; Vancso, G. J.; Soft Matter 2009, 5, 4944.

73. Wan, S.; He, D.; Yuan, Y.; Yan, Z.; Zhang, X.; Zhang, J.; Colloids Surf., B 2016, 143, 278.

74. Pang, Z.; Gao, H.; Yu, Y.; Chen, J.; Guo, L.; Ren, J.; Wen, Ziyi; Su, J.; Jiang, X.; Int. J. Pharm. 2011, 415, 1284.

75. Jiang, W.; Zhou, Y.; Yan, D.; Chem. Soc. Rev. 2015, 44, 3874.

76. Messager, L.; Gaitzsch, J.; Chierico, L.; Battaglia, G.; Curr. Opin. Pharmacol. 2014, 18, 104

77. Dieu, L-H.; Wu, D.; Palivan, C.G.; Balasubramanian, V.; Huwyler, J.; Eur. J. Pharm. Biopharm. 2014, 88, 316.

78. Li, S.; Meng, F.; Wang, Z.; Zhong, Y.; Zheng, M.; Liu, H.; Zhong, Z.; Eur. J. Pharm. Biopharm. 2012, 82, 103.

79. Nahire, R.; Haldar, M.K.; Paul, S.; Nahire, R.; Haldar, M.K.; Paul S.; Ambre, A. H.; Meghnani, V.; Layek, B.; Katti, K.S.; Gange K. N.; Singh, J.; Sarkar Kausik; Mallik, S.; Biomaterials 2014, 35, 6482.

80. Oliveira, H.; Pérez-Andrés, E.; Thevenot, J.; Sandre, O.; Berra, E.; Lecommandoux S.; J. Controlled Release 2013, 169, 165.

81. Thambi, T.; Deepagan, V. G.; Ko, H.; Lee, D. S.; Park, J. H.; J. Mater. Chem. 2012, 22, 22028

82. Pegoraro, C.; Cecchin, D.; Gracia, L. S.; Warren, N.; Madsen, J.; Armes, S. P.; Lewis, A.; MacNeil, S.; Battaglia G.; Cancer Lett. 2013, 334, 328.

83. Yu, A.; Pang, Z.; Lu, W.; Yin, Q.; Gao, H.; Jiang, X; Pharm Res. 2012, $29,83$.

84. Das, S.; Sharma, D. K.; Chakrabarty, S.; Chowdhury, A.; Sem, G. S.; Langmuir 2015, 31, 3402.

85. Zou, T.; Dembele, F.; Beugnet, A.; Sengmanivong, L.; Trepout, S.; Marco, S.; Marco, A.; Li, M.; J. Biotechnol. 2015, 214, 147.

86. Dobrunz, D.; Toma, A. C.; Tanner, P.; Pfohl, T.; Palivan, C. G.; Langmuir 2012, 28, 15889

87. Tanner, P.; Balasubramanian, V.; Palivan, C. G.; Nano Lett. 2013, 13, 2875 .

88. Sanson, C.; Diou, O.; Thevenot, J.; Ibarboure, E.; Soum, A.; Brulet, A.; Miraux, S.; Thiaudiere, E.; Tan, S.; Brisson, A.; Dupuis, V.; Sandre, O.; Lecommandoux, S.; ACS Nano 2011, 5, 1122.

89. Dionzou, M.; Morère, A.; Roux, C.; Lonetti, B.; Marty, J. D.; Mingotaud, C.; Joseph, P.; Goudounèche, D.; Payré, B.; Léonetti, M.; Mingotaud, A. F.; Soft Matter 2016, 12, 2166.

90. Meng, F.; Zhong, Z.; Feijen, J.; Biomacromolecules 2009, 10, 197.

91. Chen, W.; Meng, F.; Cheng, R.; Zhong, Z.; J. Controlled Release 2010, 142, 40.

92. Feng, A.; Yuan, J.; Macromol. Rapid Commun. 2014, 35, 767.

93. Cabane, E.; Malinova, V.; Menon, S.; Palivan, C. G.; Meier, W.; Soft Matter 2011, 7, 9167.

94. Liu, F.; Kozlovskaya, V.; Medipelli, S.; Xue, B.; Ahmad, F.; Saeed, M.; Cropek, D.; Kharlampieva, E.; Chem. Mater. 2015, 27, 7945.

95. Li, X.; Zhu, X.; Qiu, L.; Acta Biomater. 2016, 35, 269. 